\title{
Stability Analysis of State Saturation 2D Discrete Time-Delay Systems Based on F-M Model
}

\author{
Dongyan Chen and Hui Yu \\ Department of Applied Mathematics, Harbin University of Science and Technology, Harbin 150080, China \\ Correspondence should be addressed to Hui Yu; hyu_hust@163.com
}

Received 25 September 2013; Accepted 13 November 2013

Academic Editor: Hongli Dong

Copyright ( 2013 D. Chen and H. Yu. This is an open access article distributed under the Creative Commons Attribution License, which permits unrestricted use, distribution, and reproduction in any medium, provided the original work is properly cited.

\begin{abstract}
The problem of stability analysis is investigated for a class of state saturation two-dimensional (2D) discrete time-delay systems described by the Fornasini-Marchesini (F-M) model. The delay is allowed to be a bounded time-varying function. By constructing the delay-dependent 2D discrete Lyapunov functional and introducing a nonnegative scalar $\beta$, a sufficient condition is proposed to guarantee the global asymptotic stability of the addressed systems. Subsequently, the criterion is converted into the linear matrix inequalities (LMIs) which can be easily tested by using the standard numerical software. Finally, two numerical examples are given to show the effectiveness of the proposed stability criterion.
\end{abstract}

\section{Introduction}

Over the past decades, the two-dimensional (2D) systems have received considerable attention due to their extensive applications [1]. The 2D discrete-time systems have been successfully applied to many practical areas such as signal processing, linear image processing, multidimensional digital filtering, seismographic data processing, water stream heating and thermal processes. To mention a few, the problem of linear image processing has been studied in [2] for 2D discrete systems based on the Roesser model (R model). Aiming at the design of the digital filter, the 2D discrete systems based on the Fornasini-Marchesini model (F-M model) have been discussed in [3]. Recently, the modeling, calculation, and stability of 2D discrete systems have been widely studied and a large amount of results on these topics have been published; see, for example, [4-9].

Saturation, as a common and typical nonlinear constraint for practical control systems, is often encountered in various industrial systems. The phenomena of saturations if not properly handled will inevitably affect the implementations of the designed control schemes and may lead to the occurrence of the zero-input limit cycles [10-13]. Dynamical systems with saturation nonlinearities appear commonly in networks and $2 \mathrm{D}$ digital filter $[14,15]$. When designing the $2 \mathrm{D}$ digital filter by using fixed point arithmetic, saturations are introduced due to the overflow and quantization. Recently, many important results on stability analysis of $2 \mathrm{D}$ discrete F-M systems with state saturation have been reported in recent literature; see, for example, [16-21]. To be specific, by using the Lyapunov method and employing the property of matrix norm, some sufficient conditions have been presented in $[16,17]$ to guarantee the global asymptotic stability of the related systems. In [18], an internally stable condition has been given for the design of $2 \mathrm{D}$ filters. By introducing a nonnegative scalar, in $[19,20]$, the criteria based on linear matrix inequality (LMI) have been proposed to ensure the global asymptotic stability of F-M model. Recently, sufficient conditions have been derived in [21] to guarantee the global asymptotic stability of the addressed F-M systems with state saturation nonlinearities.

As well known, the time delays occur in many practical engineering systems [22-24]. The occurrence of the time delays would yield the instability of the controlled systems in some cases. So far, a great number of results have been reported; see, for example, [25-29]. In particular, considerable research attention has been devoted to the problems of stability analysis of 2D discrete time-delay systems. To be specific, by constructing an appropriate Lyapunov function and a scalar $\beta$, sufficient conditions have been proposed 
in [26] for state saturation 2D discrete time-delay systems based on the R model. In [27], the state estimation problem has been investigated for $2 \mathrm{D}$ complex networks with randomly occurring nonlinearities and probabilistic sensor delays. Accordingly, some sufficient conditions have been established such that the resulted estimation error dynamics are globally asymptotically stable in the mean square sense. To be specific, the problems of global asymptotic stability have been given in $[30,31]$ for $2 \mathrm{D}$ discrete F-M systems with state saturation and constant time delays. However, to the best of author's knowledge, there has not been much work undertaken on the global asymptotic stability for state saturation 2D discrete time-varying delay systems based on F-M model.

In this paper, we aim to investigate the problem of global asymptotic stability for 2D discrete F-M systems with state saturation and time delays. Here, the delays are assumed to be time varying with known lower and upper bounds. By constructing a delay-dependent 2D discrete Lyapunov functional and introducing a nonnegative scalar $\beta$ based on a row diagonally dominant matrix, a sufficient condition is proposed to guarantee the global asymptotic stability of the addressed systems. Subsequently, the problem of global asymptotic stability is converted into the problem of feasibility by solving the LMIs. Finally, two numerical examples are given to show the effectiveness of the proposed criterion. The main contribution of this paper lies in that new stability criterion is given for state saturation 2D discrete F-M systems with time-varying delays.

Notations. The fundamental notations used in the paper are given as follows: $I_{p}$ and 0 denote the identity matrix and zero matrix with appropriate dimensions. For a matrix $A, A^{T}$ stands for transpose of the matrix $A$. $A>0$ means that $A$ is positive definite symmetric matrix.

\section{Problem Formulation and Preliminaries}

In this paper, we consider the following $2 \mathrm{D}$ discrete $\mathrm{F}-\mathrm{M}$ system with state saturation and time-varying delays:

$$
\begin{gathered}
x(k+1, l+1)=f(y(k, l)), \\
y(k, l)=\left[y_{1}(k, l), y_{2}(k, l), \ldots, y_{n}(k, l)\right]^{T} \\
=A\left[\begin{array}{l}
x(k, l+1) \\
x(k+1, l)
\end{array}\right]+A_{d}\left[\begin{array}{c}
x\left(k-d_{h}(k), l+1\right) \\
x\left(k+1, l-d_{v}(l)\right)
\end{array}\right] \\
=A_{11} x(k, l+1)+A_{12} x(k+1, l) \\
+A_{21} x\left(k-d_{h}(k), l+1\right) \\
+A_{22} x\left(k+1, l-d_{v}(l)\right),
\end{gathered}
$$

where $x(k, l)$ is the $n$-dimensional state vector and $(k, l)$ is the 2 -dimensional time variable along the vertical and horizontal directions satisfying $k \geq 0, l \geq 0 . A=\left[A_{11}, A_{12}\right] \in R^{n \times 2 n}$, $A_{d}=\left[A_{21}, A_{22}\right] \in R^{n \times 2 n}, d_{h}(k)$ and $d_{v}(l)$ are timevarying delays along the vertical and horizontal directions, respectively. We assume $d_{h}(k)$ and $d_{v}(l)$ satisfying:

$$
h_{m} \leq d_{h}(k) \leq h_{M}, \quad v_{m} \leq d_{v}(l) \leq v_{M},
$$

where $h_{m}$ and $h_{M}$ denote the lower and upper bounds of the delay $d_{h}(k)$ along the horizontal directions, $v_{m}$ and $v_{M}$ denote the lower and upper bounds of the delay $d_{v}(l)$ along the vertical directions. Throughout this paper, the superscript $T$ to any vector (or matrix) stands for the transpose of that vector (or matrix).

The saturation nonlinearity $f(\cdot)$ is given by

$$
f(y(k, l))=\left[f_{1}\left(y_{1}(k, l)\right), f_{2}\left(y_{2}(k, l)\right), \ldots, f_{n}\left(y_{n}(k, l)\right)\right]^{T},
$$

with

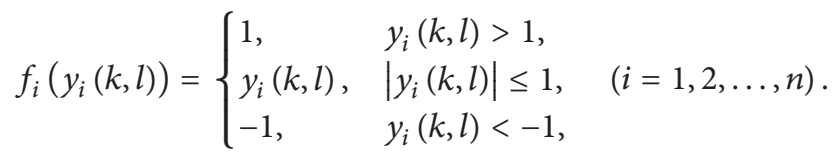

The system (1) has finite initial conditions $x(k, 0)$ and $x(0, l)$ with fixed yet nonzero values for $-h_{M} \leq k \leq K$ and $-v_{M} \leq l \leq L$, and

$$
x(k, 0)=0, \quad k \geq K ; \quad x(0, l)=0, \quad l \geq L,
$$

where $K$ and $L$ are positive integers.

To proceed, we introduce the following definitions which will be used in the subsequent derivations.

Definition 1. The origin $x=0$ of the 2D discrete time-delay system is said to be stable (in the sense of Lyapunov) if for every $\varepsilon>0$ there exists a $\delta=\delta(\varepsilon)>0$ such that

$$
\|x(k, l)\|<\varepsilon,
$$

for all $k \geq 0, l \geq 0$, whenever $\|x(k, 0)\|<\delta\left(-h_{M} \leq k \leq K\right)$ and $\|x(0, l)\|<\delta\left(-v_{M} \leq l \leq L\right)$, where $K$ and $L$ are positive integers and $\|\cdot\|$ denotes any of the equivalent norms on $R^{n}$.

Definition 2 (see [21]). The origin $x=0$ of the 2D system (1) is said to be globally asymptotically stable if the following conditions are simultaneously satisfied:

(1) system (1) is stable;

(2) every solution of system (1) tends to the origin as $k+l$ $\rightarrow \infty$; that is,

$$
\lim _{k \rightarrow \infty \text { or } l \rightarrow \infty} x(k, l)=\lim _{k+l \rightarrow \infty} x(k, l)=0 .
$$


Definition 3 (see [32]). The matrix $M$ is said to be row diagonally dominant if

$$
\left|m_{i i}\right| \geq \sum_{j=1, j \neq i}^{n}\left|m_{i j}\right|, \quad i \in[1, n] .
$$

Definition 4. For a 2D function $V(k, l)=V^{h}(k, l)+V^{v}(k, l)$, denote its difference $\Delta V(k, l)$ as

$$
\Delta V(k, l)=\Delta V^{h}(k, l)+\Delta V^{v}(k, l)
$$

where

$$
\begin{aligned}
& \Delta V^{h}(k, l)=V^{h}(k+1, l+1)-V^{h}(k, l+1), \\
& \Delta V^{v}(k, l)=V^{v}(k+1, l+1)-V^{v}(k+1, l) .
\end{aligned}
$$

\section{Main Results}

In this section, we aim to investigate the problem of stability analysis for the 2D discrete F-M system with state saturation and time-varying delays. By resorting to the LMI technique, a sufficient condition is given to ensure the global asymptotic stability of the addressed system.

Theorem 5. The origin $x=0$ of $2 D$ discrete system (1) is globally asymptotically stable if there exist matrices $P, Q$, and $M$, where $P=P_{1}+P_{2}$ and $Q=Q_{1}+Q_{2}$ with $P_{1}=\left[p_{i j}^{1}\right] \in R^{n \times n}$, $P_{2}=\left[p_{i j}^{2}\right] \in R^{n \times n}, Q_{1}=\left[q_{i j}^{1}\right] \in R^{n \times n}$, and $Q_{2}=\left[q_{i j}^{2}\right] \in R^{n \times n}$ being symmetric positive definite matrices and $M=\left[m_{i j}\right] \epsilon$ $R^{n \times n}$ is row diagonally dominant with nonnegative diagonal elements, satisfying the following matrix inequality:

$$
\left[\begin{array}{ccccc}
\Pi_{1} & M^{T} A_{11} & M^{T} A_{12} & M^{T} A_{21} & M^{T} A_{22} \\
* & \Pi_{2} & 0 & 0 & 0 \\
* & * & \Pi_{3} & 0 & 0 \\
* & * & * & -Q_{1} & 0 \\
* & * & * & * & -Q_{2}
\end{array}\right]<0
$$

with

$$
\begin{gathered}
\Pi_{1}=P_{1}+P_{2}-\left(M+M^{T}\right), \\
\Pi_{2}=Q_{1}-P_{1}+\left(h_{M}-h_{m}\right) Q_{1}, \\
\Pi_{3}=Q_{2}-P_{2}+\left(v_{M}-v_{m}\right) Q_{2} .
\end{gathered}
$$

Proof. In order to prove the global asymptotic stability of the $2 \mathrm{D}$ discrete F-M system (1), we construct the following Lyapunov functional as in [26]:

$$
V(k, l)=\sum_{i=1}^{3} V_{i}(k, l),
$$

where

$$
\begin{aligned}
V_{1}(k, l)= & V_{1}^{h}(k, l)+V_{1}^{v}(k, l) \\
= & x^{T}(k, l) P_{1} x(k, l)+x^{T}(k, l) P_{2} x(k, l), \\
V_{2}(k, l)= & V_{2}^{h}(k, l)+V_{2}^{v}(k, l) \\
= & \sum_{i=}^{k-1} x_{h}(k)(i, l) Q_{1} x(i, l) \\
& +\sum_{j=l-d_{v}(l)}^{l-1} x^{T}(k, j) Q_{2} x(k, j), \\
V_{3}(k, l)= & V_{3}^{h}(k, l)+V_{3}^{v}(k, l) \\
= & \sum_{j=-h_{M}+1}^{-h_{m}} \sum_{i=k+j}^{k-1} x^{T}(i, l) Q_{1} x(i, l) \\
& +\sum_{i=-v_{M}+1}^{-v_{m}} \sum_{j=l+i}^{l-1} x^{T}(k, j) Q_{2} x(k, j)
\end{aligned}
$$

with $P_{1}>0, P_{2}>0, Q_{1}>0$, and $Q_{2}>0$ being matrices to be determined.

According to Definition 4, the corresponding difference of $\Delta V(k, l)$ along the trajectory of system (1) can be calculated as follows:

$$
\Delta V(k, l)=\sum_{i=1}^{3} \Delta V_{i}(k, l),
$$

with

$$
\begin{aligned}
\Delta V_{1}(k, l)= & V_{1}(k+1, l+1)-x^{T}(k, l+1) P_{1} x(k, l+1) \\
& -x^{T}(k+1, l) P_{2} x(k+1, l), \\
\Delta V_{2}(k, l)= & \sum_{i=k+1-d_{h}(k+1)}^{k} x^{T}(i, l+1) Q_{1} x(i, l+1)
\end{aligned}
$$




$$
\begin{aligned}
& -\sum_{i=k-d_{h}(k)}^{k-1} x^{T}(i, l+1) Q_{1} x(i, l+1) \\
& +\sum_{j=l+1-d_{v}(l+1)}^{l} x^{T}(k+1, j) Q_{2} x(k+1, j) \\
& -\sum_{j=l-d_{v}(l)}^{l-1} x^{T}(k+1, j) Q_{2} x(k+1, j), \\
& -\sum_{j=}^{-h_{m}} \sum_{V^{+}+1}^{k} x_{i=k+j+1}^{T}(i, l+1) Q_{1} x(i, l+1) \\
& -\sum_{j=-h_{M}+1}^{-h_{m}} \sum_{i=k+j}^{k-1} x^{T}(i, l+1) Q_{1} x(i, l+1) \\
& +\sum_{i=-v_{M}+1}^{-v_{m}} \sum_{j=l+i+1}^{l} x^{T}(k+1, j) Q_{2} x(k+1, j) \\
& -\sum_{i=-v_{M}+1}^{-v_{m}} \sum_{j=l+i}^{l-1} x^{T}(k+1, j) Q_{2} x(k+1, j) .
\end{aligned}
$$

Noting (13)-(15), it can be derived that

$$
\begin{aligned}
& \Delta V_{1}(k, l)=x^{T}(k+1, l+1) P_{1} x(k+1, l+1) \\
& +x^{T}(k+1, l+1) P_{2} x(k+1, l+1) \\
& -x^{T}(k, l+1) P_{1} x(k, l+1) \\
& -x^{T}(k+1, l) P_{2} x(k+1, l), \\
& \Delta V_{2}(k, l)=x^{T}(k, l+1) Q_{1} x(k, l+1) \\
& -x^{T}\left(k-d_{h}(k), l+1\right) Q_{1} x\left(k-d_{h}(k), l+1\right) \\
& +\sum_{i=k+1-d_{h}(k+1)}^{k-1} x^{T}(i, l+1) Q_{1} x(i, l+1) \\
& -\sum_{i=k+1-d_{h}(k)}^{k-1} x^{T}(i, l+1) Q_{1} x(i, l+1) \\
& +x^{T}(k+1, l) Q_{2} x(k+1, l) \\
& -x^{T}\left(k+1, l-d_{v}(l)\right) Q_{2} x\left(k+1, l-d_{v}(l)\right) \\
& +\sum_{j=l+1-d_{v}(l+1)}^{l-1} x^{T}(k+1, j) Q_{2} x(k+1, j) \\
& -\sum_{j=l+1-d_{v}(l)}^{l-1} x^{T}(k+1, j) Q_{2} x(k+1, j)
\end{aligned}
$$$$
\leq \sum_{i=k+1-h_{M}}^{k-1} x^{T}(i, l+1) Q_{1} x(i, l+1)
$$$$
-\sum_{i=k+1-h_{m}}^{k-1} x^{T}(i, l+1) Q_{1} x(i, l+1)
$$$$
+\sum_{j=l+1-v_{M}}^{l-1} x^{T}(k+1, j) Q_{2} x(k+1, j)
$$$$
-\sum_{j=l+1-v_{m}}^{l-1} x^{T}(k+1, j) Q_{2} x(k+1, j)
$$$$
+x^{T}(k, l+1) Q_{1} x(k, l+1)
$$$$
-x^{T}\left(k-d_{h}(k), l+1\right) Q_{1} x\left(k-d_{h}(k), l+1\right)
$$$$
+x^{T}(k+1, l) Q_{2} x(k+1, l)
$$$$
-x^{T}\left(k+1, l-d_{v}(l)\right) Q_{2} x\left(k+1, l-d_{v}(l)\right)
$$$$
=\sum_{i=k+1-h_{M}}^{k-h_{m}} x^{T}(i, l+1) Q_{1} x(i, l+1)
$$$$
+\sum_{j=l+1-v_{M}}^{l-v_{m}} x^{T}(k+1, j) Q_{2} x(k+1, j)
$$$$
+x^{T}(k, l+1) Q_{1} x(k, l+1)
$$$$
-x^{T}\left(k-d_{h}(k), l+1\right) Q_{1} x\left(k-d_{h}(k), l+1\right)
$$$$
+x^{T}(k+1, l) Q_{2} x(k+1, l)
$$$$
-x^{T}\left(k+1, l-d_{v}(l)\right) Q_{2} x\left(k+1, l-d_{v}(l)\right)
$$$$
\Delta V_{3}(k, l)=\left(h_{M}-h_{m}\right) x^{T}(k, l+1) Q_{1} x(k, l+1)
$$$$
-\sum_{i=k+1-h_{M}}^{k-h_{m}} x^{T}(i, l+1) Q_{1} x(i, l+1)
$$$$
+\left(v_{M}-v_{m}\right) x^{T}(k+1, l) Q_{2} x(k+1, l)
$$$$
-\sum_{j=l+1-v_{M}}^{l-v_{m}} x^{T}(k+1, j) Q_{2} x(k+1, j) .
$$

\section{Denoting}

$$
\begin{aligned}
\varphi(k, l)=[ & f(y(k, l)), x(k, l+1), x(k+1, l), \\
& \left.x\left(k-d_{h}(k), l+1\right), x\left(k+1, l-d_{v}(l)\right)\right]^{T},
\end{aligned}
$$

we have

$$
\Delta V(k, l) \leq \varphi^{T}(k, l) \Theta \varphi(k, l),
$$


where

$$
\Theta=\left[\begin{array}{ccccc}
P_{1}+P_{2} & 0 & 0 & 0 & 0 \\
* & \left(h_{M}-h_{m}+1\right) Q_{1}-P_{1} & 0 & 0 & 0 \\
* & * & \left(v_{M}-v_{m}+1\right) Q_{2}-P_{2} & 0 & 0 \\
* & * & * & -Q_{1} & 0 \\
* & * & * & * & -Q_{2}
\end{array}\right] .
$$

Now, construct the following parameter $\beta$ :

$$
\begin{aligned}
\beta=2 \sum_{i=1}^{n}\left[y_{i}(k, l)-f_{i}\left(y_{i}(k, l)\right)\right] & \\
& \times\left[m_{i i} f_{i}\left(y_{i}(k, l)\right)+\sum_{j=1, j \neq i}^{n} m_{i j} f_{j}\left(y_{j}(k, l)\right)\right] \\
= & y^{T}(k, l) M f(y(k, l))+f^{T}(y(k, l)) M^{T} y(k, l) \\
& -f^{T}(y(k, l))\left(M+M^{T}\right) f(y(k, l)) .
\end{aligned}
$$

Let

$$
\begin{aligned}
\alpha_{i}= & {\left[y_{i}(k, l)-f_{i}\left(y_{i}(k, l)\right)\right] } \\
& \times\left[m_{i i} f_{i}\left(y_{i}(k, l)\right)+\sum_{j=1, j \neq i}^{n} m_{i j} f_{j}\left(y_{j}(k, l)\right)\right] .
\end{aligned}
$$

The saturation region can be divided into two regions, and we have

$$
\begin{gathered}
f_{i}\left(y_{i}(k, l)\right)=1, \\
y_{i}(k, l)-f_{i}\left(y_{i}(k, l)\right)>0, \text { in Region } 1, \\
f_{i}\left(y_{i}(k, l)\right)=-1, \\
y_{i}(k, l)-f_{i}\left(y_{i}(k, l)\right)<0, \text { in Region } 2 .
\end{gathered}
$$

Thus, we obtain

$$
\alpha_{i}=\left\{\begin{array}{r}
\left(y_{i}(k, l)-1\right)\left(m_{i i}+\sum_{j=1, j \neq i}^{n} m_{i j} f_{j}\left(y_{j}(k, l)\right)\right), \\
\text { in Region 1, } \\
\left(y_{i}(k, l)+1\right)\left(-m_{i i}+\sum_{j=1, j \neq i}^{n} m_{i j} f_{j}\left(y_{j}(k, l)\right)\right), \\
\text { in Region } 2 .
\end{array}\right.
$$

Subsequently, due to the property of row diagonally dominant matrix $M$, we have $\alpha_{i} \geq 0$. Furthermore, note that $\beta$ is the sum of nonnegative scalars, and hence $\beta \geq 0$. Therefore,

$$
\Delta V \leq \varphi^{T}(k, l) W \varphi(k, l)-\beta,
$$

where

$$
W=\left[\begin{array}{ccccc}
\Pi_{1} & M^{T} A_{11} & M^{T} A_{12} & M^{T} A_{21} & M^{T} A_{22} \\
* & \Pi_{2} & 0 & 0 & 0 \\
* & * & \Pi_{3} & 0 & 0 \\
* & * & * & -Q_{1} & 0 \\
* & * & * & * & -Q_{2}
\end{array}\right] .
$$

If $W<0$, then $\Delta V(k, l)<0$; that is

$$
\begin{gathered}
V^{h}(k+1, l+1)+V^{v}(k+1, l+1) \\
\leq V^{h}(k, l+1)+V^{v}(k+1, l) .
\end{gathered}
$$

Hence, for any nonnegative integer $d \geq \max \{K, L\}$, we have

$$
\begin{aligned}
\sum_{k+l=d+1} V(k, l)= & V(1, d)+V(2, d-1)+\cdots+V(d, 1) \\
\leq & V^{h}(0, d)+V^{v}(1, d-1)+V^{h}(1, d-1) \\
& +V^{v}(2, d-2)+\cdots+V^{h}(d-1,1) \\
& +V^{v}(d, 0) \\
= & \sum_{k+l=d} V(k, l) .
\end{aligned}
$$

It is clear that the sum of the Lyapunov functional is a decreasing function along the state trajectories of system (1). Then, noting the initial condition $x(0, d)=x(d, 0)=0$, we have

$$
\lim _{k \rightarrow \infty \text { or } l \rightarrow \infty} x(k, l)=\lim _{k+l \rightarrow \infty} x(k, l)=0 .
$$

Summarizing the above discussions, it can be shown that (11) is a sufficient condition which ensures the global asymptotic stability of system (1). This completes the proof of Theorem 5 .

As special cases, if there is no time delay in system (1), that is, $A_{21}=0$ and $A_{22}=0$, or the time delays are constant, then we can have the following corollaries.

Corollary 6. Consider the system (1) without time delay. If there exist matrices $P$ and $M$, with $P=P_{1}+P_{2}, P_{1}=\left[p_{i j}^{1}\right] \epsilon$ $R^{n \times n}$, and $P_{2}=\left[p_{i j}^{2}\right] \in R^{n \times n}$ being symmetric positive definite matrices and $M=\left[m_{i j}\right] \in R^{n \times n}$ is row diagonally dominant 
with nonnegative diagonal elements, satisfying the following matrix inequality:

$$
\left[\begin{array}{ccc}
P-\left(M+M^{T}\right) & M^{T} A_{11} & M^{T} A_{12} \\
* & -P_{1} & 0 \\
* & 0 & -P_{2}
\end{array}\right]<0
$$

then the origin $x=0$ of $2 D$ discrete system (1) without time delay is globally asymptotically stable.
Corollary 7. Consider the constant time delay $d_{h}(k)=d_{h}$, $d_{v}(l)=d_{v}$. If there exist matrices $P, Q$, and $M$, where $P=$ $P_{1}+P_{2}$ and $Q=Q_{1}+Q_{2}$, with $P_{1}=\left[p_{i j}^{1}\right] \in R^{n \times n}, P_{2}=$ $\left[p_{i j}^{2}\right] \in R^{n \times n}, Q_{1}=\left[q_{i j}^{1}\right] \in R^{n \times n}$, and $Q_{2}=\left[q_{i j}^{2}\right] \in R^{n \times n}$ being symmetric positive definite matrices and $M=\left[m_{i j}\right] \in R^{n \times n}$ is row diagonally dominant with nonnegative diagonal elements, satisfying the following matrix inequality:

$$
\left[\begin{array}{ccccc}
P_{1}+P_{2}-\left(M+M^{T}\right) & M^{T} A_{11} & M^{T} A_{12} & M^{T} A_{21} & M^{T} A_{22} \\
* & Q_{1}-P_{1} & 0 & 0 & 0 \\
* & * & Q_{2}-P_{2} & 0 & 0 \\
* & * & * & -Q_{1} & 0 \\
* & * & * & * & -Q_{2}
\end{array}\right]<0
$$

then the origin $x=0$ of $2 D$ discrete system (1) with constant time delay is globally asymptotically stable.

Remark 8 . Note that the problem of global asymptotic stability has been investigated in [33] for state saturation 2D discrete system without time delay. Accordingly, a stability criterion has been derived in [33] but a positive diagonally matrix is needed to be searched. It can be easily seen that the stability condition in [33] is a special case of (30) in Corollary 6. Therefore, the stability condition proposed in this paper is less conservative than the one in [33]. Meanwhile, note that a stability condition has been proposed in [21] where an unknown matrix $G$ must be given firstly. The values of $G$ must take specific values. Compared with the results in [21], we only need to find matrix $M$. It concludes that the stability condition in [21] is more conservative than our result.

Remark 9. It is worth mentioning that the delay-fractioning approach has been employed in $[34,35]$ to reduce the conservativeness of the time delay. It has been shown that the developed approach performs well when dealing with the time delay compared with other methods. Accordingly, some effective SMC/SMO schemes based on the delay-fractioning idea have been proposed for discrete time-delay nonlinear stochastic systems with randomly occurring incomplete information. Motivated by the results in $[34,35]$, we are now researching into the stability criterion based on the delayfractioning approach for the state saturation $2 \mathrm{D}$ discrete time-delay systems. The corresponding results will appear in the near future. Moreover, note that the recursive filters have been designed in [36-38] for time-varying networked nonlinear systems with missing measurements. It is also interesting to consider the analysis and synthesis of $2 \mathrm{D}$ discrete nonlinear systems with state saturations and missing measurements.

Remark 10. It is worth noting that the conditions in Theorem 5 are not strict LMI due to the fact that the matrix $M=\left[m_{i j}\right] \in R^{n \times n}$ is row diagonally dominant with nonnegative diagonal elements. Hence, the results of Theorem 5 and Corollary 6 are not convex which lead to the computational difficulties. In the following, an alternative approach is developed to deal with the nonconvex problem.

Let $e_{l}$ be $n$-dimensional column vectors in which the $l$ th element is 1 and other elements are 0 . Let $Y_{l}$ be the set of $n$-dimensional column vectors in which the $l$ th element is -1 and other elements are either 1 or -1 and $Y_{l s} \in Y_{l}$ $\left(s \in\left[1,2^{n-1}\right]\right)$. Then, the condition where matrix $M=$ $\left[m_{i j}\right] \in R^{n \times n}$ is row diagonally dominant and the diagonal is composed of nonnegative elements can be equivalently converted into the following LMIs:

$$
e_{l}^{T} M Y_{l s}<0, \quad l=1,2, \ldots, m+n ; s \in\left[1,2^{m+n-1}\right]
$$

Together with (11) and (32), we can see that the proposed stability condition is a convex one and then can be easily solved by using the standard numerical software.

\section{Numerical Examples}

In this section, two numerical examples are given here to illustrate the effectiveness of the main results.

Example 1. Consider the state saturation 2D discrete timedelay system (1) with

$$
\begin{aligned}
A_{11} & =\left[\begin{array}{cccc}
1.678 & 0.2853 & -0.2432 & -0.1246 \\
-0.84 & 0.23 & -0.1 & 1.45 \\
0.4 & 0.0217 & 0.1785 & 0.1662 \\
0.0245 & -0.00884 & 0.0321 & -0.2428
\end{array}\right], \\
A_{12} & =\left[\begin{array}{cccc}
-0.495 & -0.687 & -0.030 & -0.3013 \\
-0.1630 & -0.4817 & -0.993 & 0.1814 \\
0.2842 & 0.1790 & -0.5551 & 0.0799 \\
-0.2112 & 0.1887 & 0.0895 & 0.5604
\end{array}\right],
\end{aligned}
$$




$$
\begin{aligned}
A_{21} & =\left[\begin{array}{cccc}
-9.987 & -0.2 & 0 & 0 \\
0 & -0.15 & 0 & -0.1 \\
0 & 0 & -0.78 & 0 \\
-0.23 & 0 & 0 & -0.98
\end{array}\right], \\
A_{22} & =\left[\begin{array}{cccc}
5.763 & -0.067 & 0 & 0.04 \\
-2.314 & 0.342 & 0.098 & -5.78 \\
4.56 & 0.001 & -0.98 & 0.231 \\
0.23 & -0.76 & 0.043 & 8.6
\end{array}\right] .
\end{aligned}
$$

The lower and upper bounds are given by $h_{M}=3, h_{m}=1$, $v_{M}=4$, and $v_{m}=2$.

Solving the inequalities (11) and (32) in the Matlab environment, we can obtain

$$
\begin{aligned}
P_{1} & =\left[\begin{array}{cccc}
3.0067 & -0.0360 & -0.0043 & 0.0125 \\
-0.0360 & 3.1420 & 0.1635 & 0.2573 \\
-0.0043 & 0.1635 & 2.9443 & 0.1253 \\
0.0125 & 0.2573 & 0.1253 & 2.9694
\end{array}\right], \\
P_{2} & =\left[\begin{array}{cccc}
2.9848 & -0.0225 & -0.0006 & 0.0463 \\
-0.0225 & 3.1494 & 0.1757 & 0.2382 \\
-0.0006 & 0.1757 & 3.0049 & 0.0990 \\
0.0463 & 0.2382 & 0.0990 & 2.9051
\end{array}\right], \\
Q_{1} & =\left[\begin{array}{cccc}
0.5521 & -0.0048 & -0.0017 & 0.0417 \\
-0.0048 & 0.6168 & 0.0461 & 0.0573 \\
-0.0017 & 0.0461 & 0.5631 & 0.0356 \\
0.0417 & 0.0573 & 0.0356 & 0.4917
\end{array}\right], \\
Q_{2} & =\left[\begin{array}{cccc}
0.5676 & -0.0144 & -0.0043 & 0.0175 \\
-0.0144 & 0.6115 & 0.0374 & 0.0709 \\
-0.0043 & 0.0374 & 0.5198 & 0.0545 \\
0.0175 & 0.0709 & 0.0545 & 0.5376
\end{array}\right], \\
M & =\left[\begin{array}{cccc}
0.2812 & 0.0241 & -0.0475 & -0.0020 \\
0.0353 & 0.6877 & 0.0609 & 0.2516 \\
-0.0769 & 0.0654 & 0.7074 & 0.0128 \\
0.0027 & 0.1886 & -0.0018 & 0.5205
\end{array}\right] .
\end{aligned}
$$

Then, we can see that there exist the required matrices $P_{1}$, $P_{2}, Q_{1}, Q_{2}$, and $M$ satisfying LMIs (11) and (32). As such, according to Theorem 5, the origin $x=0$ of system (1) is globally asymptotically stable which confirms the feasibility of the proposed main results.

Example 2. Consider a 2D system described by (1) without time delay. The system parameters are given as

$$
\begin{gathered}
A_{11}=\left[\begin{array}{cc}
1.2 & -2.8 \\
0.1 & 0
\end{array}\right], \\
A_{12}=\left[\begin{array}{ll}
0 & 0.01 \\
0 & 0.02
\end{array}\right] .
\end{gathered}
$$

By using the Matlab LMI Toolbox, it can be easily verified that the following feasible solutions can be obtained

$$
\begin{gathered}
P_{1}=\left[\begin{array}{cc}
1421.7 & -203.9 \\
-203.9 & 1698.1
\end{array}\right], \quad P_{2}=\left[\begin{array}{cc}
1321 & 25 \\
25 & 1167.5
\end{array}\right], \\
M=\left[\begin{array}{cc}
434.848 & -11.3033 \\
-9.1992 & 818.5481
\end{array}\right] .
\end{gathered}
$$

That is, there exist the required matrices $P_{1}, P_{2}$, and $M$ satisfying LMI (30). According to Corollary 6, the origin of system (1) is globally asymptotically stable which confirms the effectiveness of the presented results. However, it can be tested that the conditions in [33] are infeasible. Therefore, the result in our paper is less conservative than the one in [33].

\section{Conclusions}

In this paper, we have discussed the problem of global asymptotic stability for 2D discrete F-M systems with state saturation and time-varying delays. By constructing the $2 \mathrm{D}$ discrete-time Lyapunov functional, a new stability criterion has been established to ensure that the addressed system is globally asymptotically stable. The proposed stability criterion is in terms of the LMIs which can be easily tested by using the Matlab matrix toolbox. Two numerical examples have been given to demonstrate the feasibility of the proposed stability condition. It is worth mentioning that the construction of the scalar $\beta$ has taken full effects from time delays with hope to reduce the conservativeness. One of the future research topics would be the extension of the proposed main results to more general state saturation $2 \mathrm{D}$ discrete systems as in [39-41] with network-induced phenomena.

\section{Acknowledgments}

This work was supported in part by the Science Research Project of Abroad Scholars of Educational Commission of Heilongjiang Province under Grant 1152hq09 and the National Natural Science Foundation of China under Grants 11271103 and 11301118.

\section{References}

[1] C. E. de Souza, L. Xie, and D. F. Coutinho, "Robust filtering for 2-D discrete-time linear systems with convex-bounded parameter uncertainty," Automatica, vol. 46, no. 4, pp. 673-681, 2010.

[2] R. P. Roesser, "A discrete state-space model for linear image processing," IEEE Transactions on Automatic Control, vol. 20, no. 1, pp. 1-10, 1975.

[3] E. Fornasini and G. Marchesini, "State-space realization theory of two-dimensional filters," IEEE Transactions on Automatic Control, vol. 21, no. 4, pp. 484-492, 1976.

[4] N. G. El-Agizi and M. M. Fahmy, "Two-dimensional digital filters with no overflow oscillations," IEEE Transactions on Acoustics, Speech and Signal Processing, vol. 27, no. 5, pp. 465469, 1979.

[5] H. Gao, J. Lam, S. Xu, and C. Wang, "Stability and stabilization of uncertain 2-D discrete systems with stochastic perturbation," 
Multidimensional Systems and Signal Processing, vol. 16, no. 1, pp. 85-106, 2005.

[6] H. Gao, J. Lam, C. Wang, and S. Xu, " $H_{\infty}$ model reduction for uncertain two-dimensional discrete systems," Optimal Control Applications \& Methods, vol. 26, no. 4, pp. 199-227, 2005.

[7] P. Agathoklis, "Lower bounds for the stability margin of discrete two-dimensional systems based on the two-dimensional Lyapunov equation," IEEE Transactions on Circuits and Systems, vol. 35, no. 6, pp. 745-749, 1988.

[8] M. G. B. Sumanasena and P. H. Bauer, "Realization using the Fornasini-Marchesini model for implementations in distributed grid sensor networks," IEEE Transactions on Circuits and Systems I: Regular Papers, vol. 58, no. 11, pp. 2708-2717, 2011.

[9] X. Li and H. Gao, "Robust finite frequency $H_{\infty}$ filtering for uncertain 2-D systems: the FM model case," Automatica, vol. 49, no. 8, pp. 2446-2452, 2013.

[10] T. A. C. M. Claasen, W. F. G. Mecklenbräuker, and J. B. H. Peek, "Effects of quantization and overflow in recursive digital filters," IEEE Transactions on Acoustics, Speech and Signal Processing, vol. 24 , no. 6 , pp. 517-529, 1976.

[11] D. Chen, S. Li, and Y. Shi, "The practical stabilization for a class of networked systems with actuator saturation and input additive disturbances," Mathematical Problems in Engineering, vol. 2012, Article ID 326876, 19 pages, 2012.

[12] J. Hu, Z. Wang, H. Gao, and L. K. Stergioulas, "Probabilityguaranteed $H_{\infty}$ finite-horizon filtering for a class of nonlinear time-varying systems with sensor saturations," Systems \& Control Letters, vol. 61, no. 4, pp. 477-484, 2012.

[13] Z. Wang, B. Shen, and X. Liu, " $H_{\infty}$ filtering with randomly occurring sensor saturations and missing measurements," Automatica, vol. 48, no. 3, pp. 556-562, 2012.

[14] H. Kar and V. Singh, "Stability analysis of 2-D digital filters with saturation arithmetic: an LMI approach," IEEE Transactions on Signal Processing, vol. 53, no. 6, pp. 2267-2271, 2005.

[15] V. Singh, "Robust stability of 2-D digital filters employing saturation," IEEE Signal Processing Letters, vol. 12, no. 2, pp. 142$145,2005$.

[16] D. R. Liu and A. N. Michel, "Stability analysis of statespace realizations for two-dimensional filters with overflow nonlinearities," IEEE Transactions on Circuits and Systems I: Fundamental Theory and Applications, vol. 41, no. 2, pp. 127-137, 1994.

[17] D. Liu, "Lyapunov stability of two-dimensional digital filters with overflow nonlinearities," IEEE Transactions on Circuits and Systems I: Fundamental Theory and Applications, vol. 45, no. 5, pp. 574-577, 1998.

[18] E. Fornasini and G. Marchesini, "On the internal stability of two-dimensional filters," IEEE Transactions on Automatic Control, vol. 24, no. 1, pp. 129-130, 1979.

[19] H. Kar and V. Singh, "Stability analysis of 2-D digital filters described by the Fornasini-Marchesini second model using overflow nonlinearities," IEEE Transactions on Circuits and Systems I: Fundamental Theory and Applications, vol. 48, no. 5, pp. 612-617, 2001.

[20] H. Kar and V. Singh, "Stability analysis of 1-D and 2-D fixed-point state-space digital filters using any combination of overflow and quantization nonlinearities," IEEE Transactions on Signal Processing, vol. 49, no. 5, pp. 1097-1105, 2001.

[21] V. Singh, "Stability analysis of 2-D discrete systems described by the Fornasini-Marchesini second model with state saturation," IEEE Transactions on Circuits and Systems II : Express Briefs, vol. 55, no. 8, pp. 793-796, 2008.
[22] Z. Wang, Y. Liu, and X. Liu, "Exponential stabilization of a class of stochastic system with Markovian jump parameters and mode-dependent mixed time-delays," IEEE Transactions on Automatic Control, vol. 55, no. 7, pp. 1656-1662, 2010.

[23] H. Dong, Z. Wang, and H. Gao, "Distributed $H_{\infty}$ filtering for a class of Markovian jump nonlinear time-delay systems overlossy sensor networks," IEEE Transactions on Industrial Electronics, vol. 60, no. 10, pp. 4665-4672, 2013.

[24] J. Hu, Z. Wang, H. Gao, and L. K. Stergioulas, "Robust $H_{\infty}$ sliding mode control for discrete time-delay systems with stochastic nonlinearities," Journal of the Franklin Institute, vol. 349, no. 4, pp. 1459-1479, 2012.

[25] B. Shen, S. X. Ding, and Z. Wang, "Finite-horizon $H_{\infty}$ fault estimation for linear discrete time-varying systems with delayed measurements," Automatica, vol. 49, no. 1, pp. 293-296, 2013.

[26] S.-F. Chen, "Delay-dependent stability for 2D systems with time-varying delay subject to state saturation in the Roesser model," Applied Mathematics and Computation, vol. 216, no. 9, pp. 2613-2622, 2010.

[27] J. Liang, Z. Wang, and X. Liu, "State estimation for twodimensional complex networks with randomly occurring nonlinearities and randomly varying sensor delays," International Journal of Robust and Nonlinear Control.

[28] J. Hu, Z. Wang, B. Shen, and H. Gao, "Gain-constrained recursive filtering with stochastic nonlinearities and probabilistic sensor delays," IEEE Transactions on Signal Processing, vol. 61, no. 5, pp. 1230-1238, 2013.

[29] Z. Wang, B. Shen, H. Shu, and G. Wei, "Quantized $H_{\infty}$ control for nonlinear stochastic time-delay systems with missing measurements," IEEE Transactions on Automatic Control, vol. 57, no. 6, pp. 1431-1444, 2012.

[30] S.-F. Chen, "Stability analysis of 2-D state-delayed systems with saturation nonlinearities," in Proceedings of the 7th Asian Control Conference (ASCC '09), pp. 412-417, Hong Kong, China, August 2009.

[31] A. Dey and H. Kar, "An LMI based criterion for the global asymptotic stability of 2-D discrete state-delayed systems with saturation nonlinearities," Digital Signal Processing, vol. 22, no. 4, pp. 633-639, 2012.

[32] H. Fang and Z. Lin, "Stability analysis for linear systems under state constraints," IEEE Transactions on Automatic Control, vol. 49, no. 6, pp. 950-955, 2004.

[33] T. Hinamoto, "Stability of 2-D discrete systems described by the Fornasini-Marchesini second model," IEEE Transactions on Circuits and Systems I: Fundamental Theory and Applications, vol. 44, no. 3, pp. 254-257, 1997.

[34] J. Hu, Z. Wang, Y. Niu, and L. K. Stergioulas, " $H_{\infty}$ sliding mode observer design for a class of nonlinear discrete time-delay systems: a delay-fractioning approach," International Journal of Robust and Nonlinear Control, vol. 22, no. 16, pp. 1806-1826, 2012.

[35] J. Hu, Z. Wang, H. Gao, and L. K. Stergioulas, "Robust sliding mode control for discrete stochastic systems with mixed time delays, randomly occurring uncertainties, and randomly occurring nonlinearities," IEEE Transactions on Industrial Electronics, vol. 59, no. 7, pp. 3008-3015, 2012.

[36] J. Hu, Z. Wang, H. Gao, and L. K. Stergioulas, "Extended Kalman filtering with stochastic nonlinearities and multiple missing measurements," Automatica, vol. 48, no. 9, pp. 20072015, 2012.

[37] H. Dong, Z. Wang, and H. Gao, "Distributed filtering for a class of time-varying systems over sensor networks with quantization 
errors and successive packet dropouts," IEEE Transactions on Signal Processing, vol. 60, no. 6, pp. 3164-3173, 2012.

[38] J. Hu, Z. Wang, and H. Gao, "Recursive filtering with random parameter matrices, multiple fading measurements and correlated noises," Automatica, vol. 49, no. 11, pp. 3440-3448, 2013.

[39] Z. Wang, D. Ding, H. Dong, and H. Shu, " $H_{\infty}$ consensus control for multi-agent systems with missing measurements: the finitehorizon case," Systems and Control Letters, vol. 62, no. 10, pp. 827-836, 2013.

[40] J. Hu, Z. Wang, B. Shen, and H. Gao, "Quantised recursive filtering for a class of nonlinear systems with multiplicative noises and missing measurements," International Journal of Control, vol. 86, no. 4, pp. 650-663, 2013.

[41] Z. Wang, H. Dong, B. Shen, and H. Gao, "Finite-horizon $H_{\infty}$ filtering with missing measurements and quantization effects," IEEE Transactions on Automatic Control, vol. 58, no. 7, pp. 17071718, 2013. 


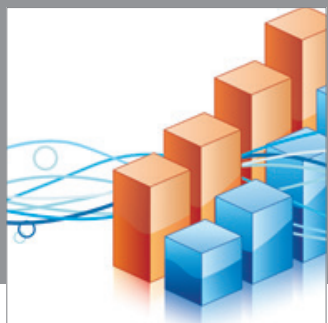

Advances in

Operations Research

mansans

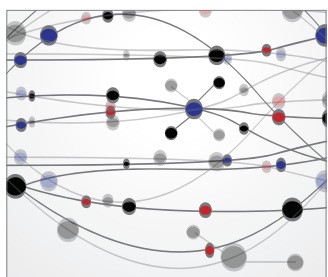

The Scientific World Journal
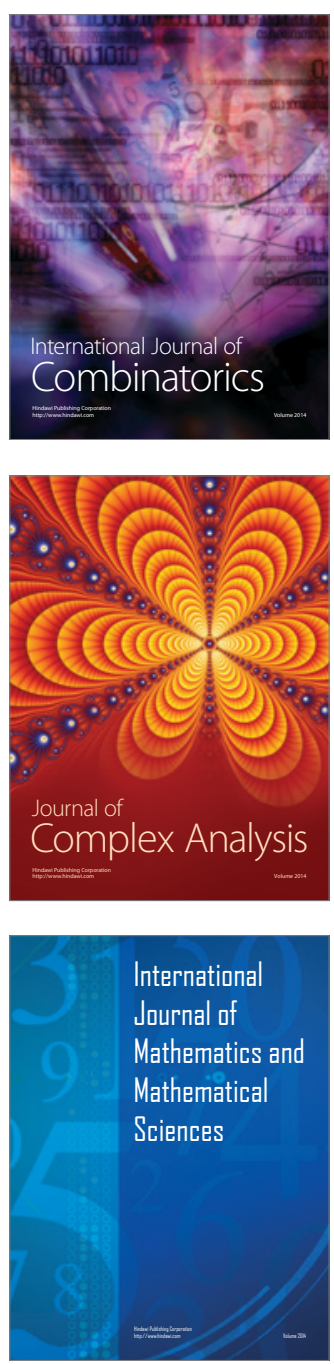
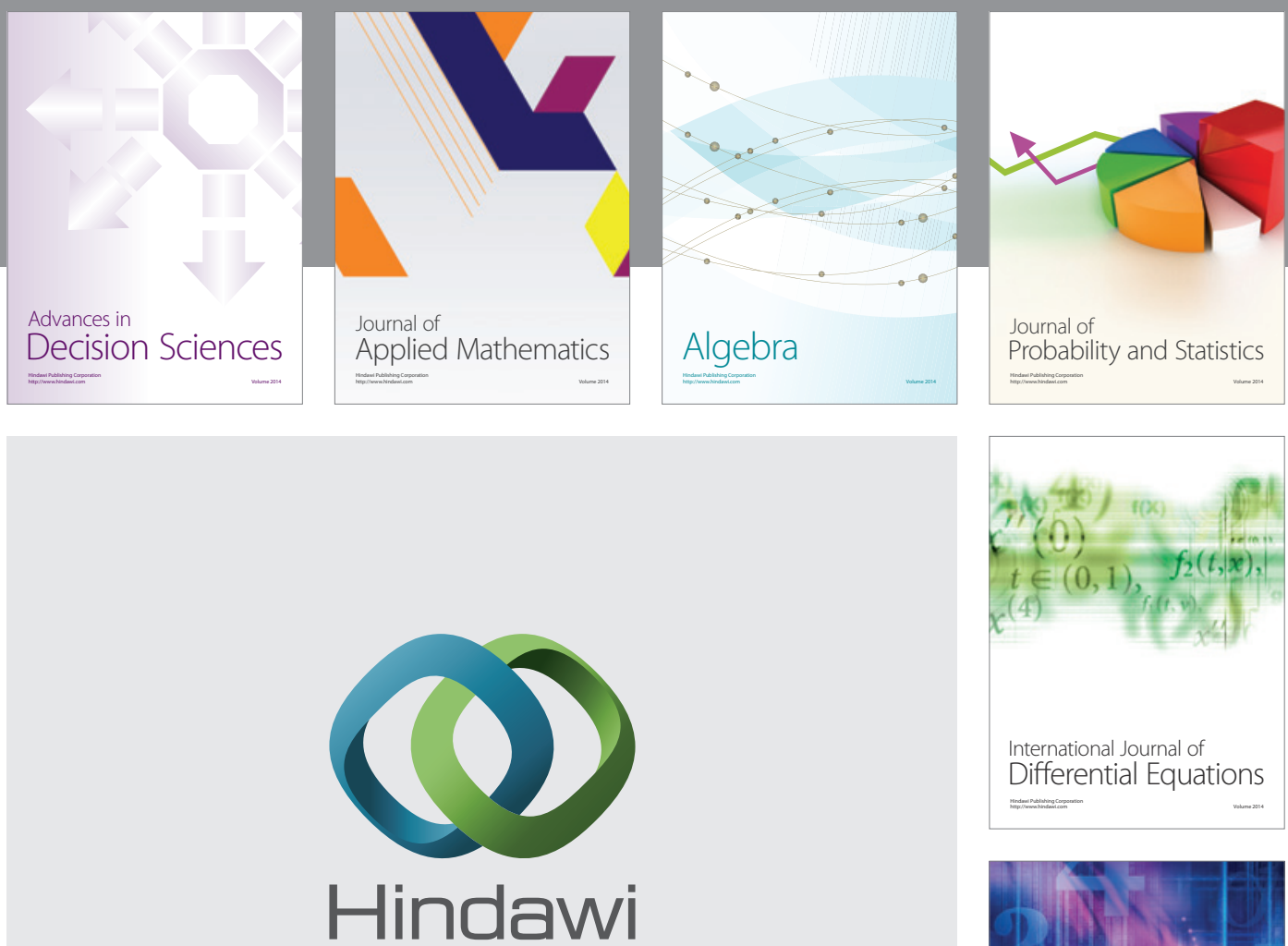

Submit your manuscripts at http://www.hindawi.com
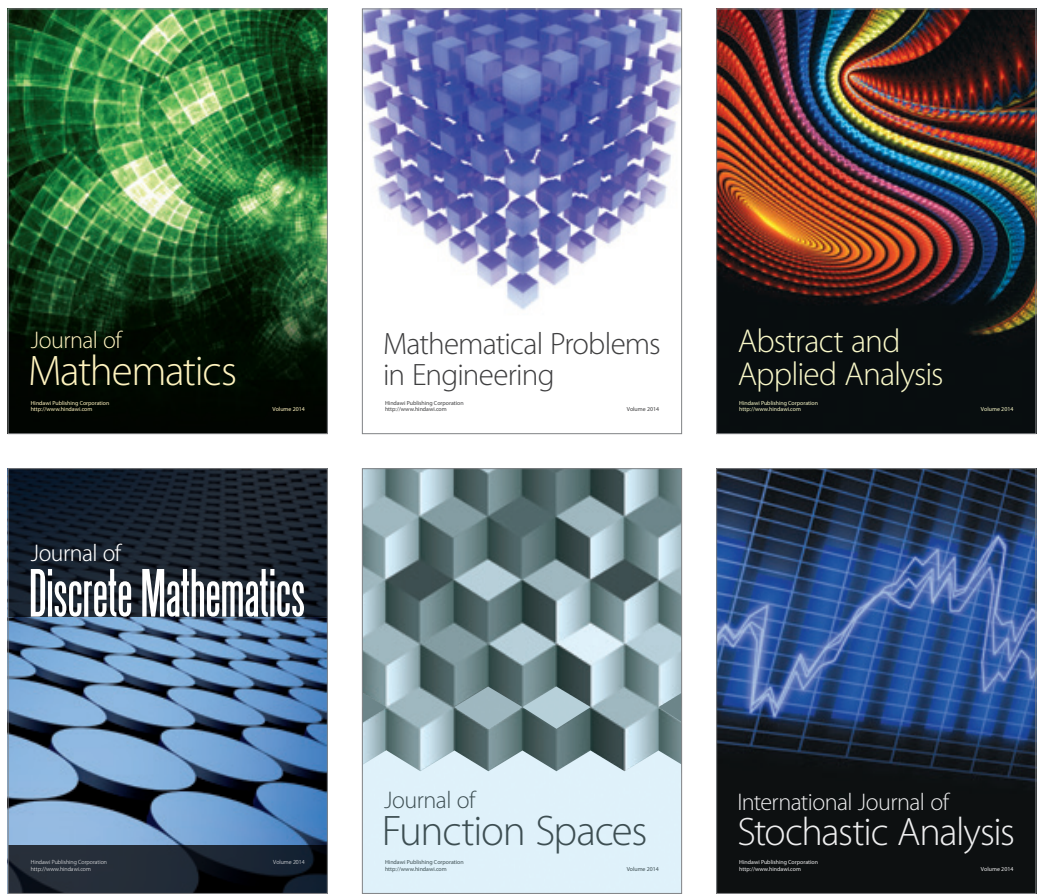

Journal of

Function Spaces

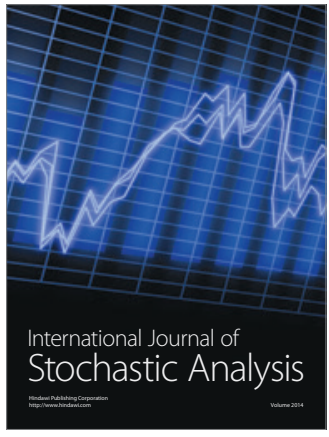

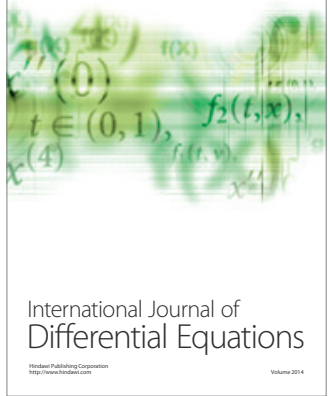
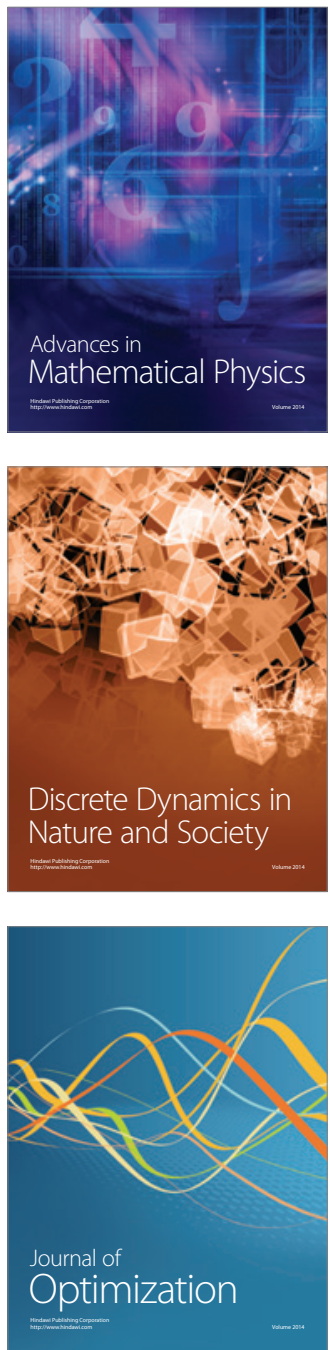